\title{
Extracción de algas en Pisco: Desafíos, oportunidades, adaptación y perspectivas futuras
}

\begin{abstract}
RESUMEN
La extracción de algas marinas es una actividad que, en la provincia de Pisco, genera empleo para más de cuatrocientas familias que extraen este recurso, a su vez es un elemento fundamental en el ecosistema marino que estaría siendo afectado por la contaminación de este espacio. A fin de identificar la problemática integral vinculada a este recurso y a su explotación así como los impactos socioeconómicos existentes y proponer alternativas, se realizó un trabajo participativo universidad - comunidad que devino en la realización de un seminario - taller a partir del cual se formularon propuestas.

Palabras clave: extracción de algas, contaminación del espacio marino, interacción universidad - comunidad.
\end{abstract}

Extraction of Seaweed in Pisco: Challenges, OPportunities, ADAPTATION AND FUTURE PERSPECTIVES

\section{ABSTRACT}

The extraction of seaweed is an activity that, in Pis$\mathrm{co}$, generates payments for more than four hundred families than they extract this resource, that is also a fundamental element in the marine ecosystem and which would be being affected by the contamination of this space. In order to identify the integral problem linked to this resource and its operation, and the socio-economic impacts and to propose alternatives, a participating work was made during the accomplishment of a workshop from which it was formulated proposals.

Key words: seaweed extraction, marine space pollution, university - community interaction

\section{INTRODUCCIÓN}

El distrito de San Andrés está ubicado a $4,9 \mathrm{~km}$. del puerto de Pisco en el departamento de Ica. En el 2007 (INEl, 2007) su población era de 13151 personas, que en su mayoría se dedican a la pesca artesanal. Cabe recalcar que el departamento de Ica cuenta con 17 plantas de harina de pescado y 11 plantas de enlatados, distribuidas en su litoral. De igual importancia es la presencia de Pluspetrol, una compañía de gas y líquidos asociados que utiliza gas proveniente de la región de Camisea. San Andrés no solo se encuentra ubicado en las cercanías de plantas de harina de pescado y de Pluspetrol, sino también en las cercanías de las Islas Ballestas y de la Reserva Nacional de Paracas. Estas últimas son puntos de gran atractivo turístico en Pisco donde se pueden observar impresionantes formaciones rocosas que son hábitat de lobos marinos, pingüinos, flamencos, etc.

Las actividades industriales así como el crecimiento poblacional de la zona han provocado el incremento de contaminantes en la mencionada área del litoral pisqueño. Por ejemplo, el Instituto del Mar de Perú (Imarpe) en enero de 1999 detectó concentraciones de cadmio de alrededor de $2 \mathrm{ug} / \mathrm{g}$. Imarpe también concluyó otros estudios y sugirió la optimización de algunas técnicas en las plantas de harina de pescado. Por ejemplo, Imarpe ha sugerido el uso de agua dulce para el procesamiento de pescado y el reciclaje de esta agua. Asimismo, sugirió monitorear y analizar los efluentes de las plantas de harina de pescado (sólidos suspendidos totales, aceites y grasas, y demanda bioquímica de oxígeno). Para mitigar el impacto de las actividades de las plantas de harina de pescado, la Asociación de Productores de Harina de Pescado (Apropisco) ha puesto en operación un emisor submarino de 18 pulgadas de diámetro interior que descarga efluentes de las plantas de harina de pescado a aproximadamente $12 \mathrm{~km}$ de la bahía.

Otra de las actividades económicas que también se desarrollan en San Andrés es la extracción de algas. En el Perú, esta actividad es relativamente nueva y se realiza en forma artesanal. Las algas son vendidas a diferentes mercados tanto en el Perú como en el extranjero. En el mercado peruano las algas son utilizadas en la elaboración de shampoo, jabones y otros productos de aseo personal. En el extranjero, los usos varían e incluyen el

\footnotetext{
Msc, estudiante de doctorado de la Memorial University of Newfoundland - Canada. E-mail:claudiavivanco@mun.ca

2 Dsc, docente del Departamento de Ingeniería de la PUCP. E-mail: jcalvarez@pucp.edu.pe

3 DSc, profesora de Memorial University of Newfoundland - Canada. E-mail: kvodden@mun.ca
} 
consumo humano, fabricación de productos de aseo personal, insumos para la industria farmacológica, etc. Estos mercados tienen preferencias variadas, por ejemplo, los mercados asiáticos prefieren algas rosadas o rojizas, mientras que los mercados peruanos prefieren algas verdes. Otros mercados prefieren algas de color amarillo. Estos diversos colores se pueden adquirir a través de diferentes procesos en los que se pueden emplear algunas substancias químicas que facilitan el proceso de secado. Sin embargo, el uso de estos químicos impide la obtención de certificación orgánica. El mercado nacional y extranjero no solo difiere en las preferencias de color de algas, sino también en el precio. Por ejemplo, los algueros venden un kilogramo de algas en Pisco a un sol, mientras que en Lima las algas se venden a dos soles y en el mercado extranjero el precio es de alrededor veinticuatro soles.

Estas asociaciones cuentan con instalaciones artesanales donde se procesan algas. La extracción y procesamiento de algas es una actividad laboral atractiva en San Andrés debido a que emplea alrededor de veinte algueros en cada instalación. Desde el 2003 otros distritos en Pisco han experimentado el cultivo artesanal de algas. Sin embargo, estos experimentos no han sido efectivos en su totalidad. La ausencia de instalaciones de procesamiento adecuadas, que cumplan con los requisitos de seguridad, limita la eficiencia de estos experimentos. Además, el procesamiento artesanal de algas, en la mayor parte de los casos, no cumple con los estándares del mercado nacional o extranjero. Es necesario promover estudios de investigación que determinen los factores óptimos para el cultivo de estas algas.

Además del distrito de San Andrés, que cuenta con dos asociaciones de extractores de algas, en el vecino distrito de San Clemente también existen dos asociaciones, con las cuales mantienen coordinaciones.

El trabajo participativo realizado tuvo como objetivo encuestar a los algueros en temas relacionados con 1) desafíos y oportunidades que la extracción de algas trae consigo, 2) cambios ambientales observados en la bahía de Paracas, 3) posibles medidas de adaptación para mitigar los efectos ambientales adversos, 4) acciones a futuro en la industria de las algas.

La encuesta consistió en una serie de preguntas de los mencionados temas. Algunas preguntas fueron de desarrollo, en las cuales se pidió que los algueros escribieran sus respuestas. Mientras que otras preguntas -en la misma encuesta- consistieron en escoger y marcar las alternativas que ellos consi- deraron convenientes. A través de esta encuesta se logró determinar el grado de conocimiento ambiental de los algueros en la bahía de Pisco.

El resultado de la encuesta se presenta y analiza en este trabajo, el cual resume la exposición e intercambio de ideas con respecto a los desafíos, oportunidades, adaptación y perspectivas futuras concernientes a la extracción de algas en Pisco, Perú.

\section{INVESTIGACIÓN PARTICIPATIVA SOBRE LA PROBLEMÁTICA DE LAS ALGAS EN PISCO}

\section{Agenda del Taller}

El taller Extracción de algas en la bahía de Paracas y Pisco: Desafíos, Oportunidades, Adaptación y Perspectivas Futuras realizado el 6 de junio del 2009 , siguió la agenda que se presenta a continuación: bienvenida y explicación acerca de los objetivos del taller, llenado de una encuesta en forma anónima, diálogo e intercambio de ideas acerca de las posibles oportunidades en la extracción de algas, diálogo acerca de los desafíos en la extracción de algas y posibles soluciones, diálogo e intercambio de ideas acerca de cambios ambientales observados en la bahía de Paracas, diálogo e intercambio de ideas acerca de acciones futuras en el desarrollo de actividades de extracción de algas, clausura y agradecimientos.

\section{Encuesta y Resultados de la Encuesta}

Al taller asistieron miembros de las asociaciones San Andrés y Señor del Mar de San Andrés y también de la Asociación Beatita de Humay de San Clemente.

Considerando que en ambos distritos aproximadamente 400 familias se dedican a esta actividad -y dada la participación de 20 extractores en representación de sus respectivas familias- el tamaño de la muestra representa un $5 \%$.

En esta parte se muestran las preguntas que formaron parte de la encuesta a los algueros, así como las respuestas a estas preguntas. El cuestionario fue formulado después de una serie de entrevistas previas a científicos (Concytec, Imarpe), profesores de universidades (UNALM, UNMSM, Universidad Juan Luis Gonzaga de Ica) y a los algueros de las mencionadas asociaciones. Las respuestas que acumularon más votos se encuentran resaltadas en negrita. De la misma manera, las respuestas a las preguntas de desarrollo han sido transcritas literalmente y se encuentran resaltadas en negrita. 
1. Desafíos y oportunidades.

1.1 ¿Cuáles son las mayores dificultades para la actividad de extracción/ cultivo de algas en Pisco/ Paracas?

Escoger y marcar (X) las tres (3) más importantes.

\begin{tabular}{|l|c|}
\hline Contaminación & $\mathbf{2 3}$ \\
\hline Hay pocas algas & 12 \\
\hline Extracción exagerada de algas & 2 \\
\hline $\begin{array}{l}\text { Áreas restringidas para la actividad y } \\
\text { conflicto sobre el espacio marino }\end{array}$ & 10 \\
\hline Entrada de aguas calientes a la zona & 3 \\
\hline Falta de planificación de la extracción de algas & 6 \\
\hline Falta de conocimiento y entrenamiento & 1 \\
\hline Veda de algas & 3 \\
\hline Falta de procesamiento industrial & 9 \\
\hline Otra ... & 1 \\
\hline
\end{tabular}

1.2 ¿Qué oportunidades o tendencias positivas usted ve para la actividad de extracción/ cultivo de algas en Pisco/Paracas?

Escoger y marcar $(X)$ las tres (3) más importantes.

\begin{tabular}{|l|c|}
\hline $\begin{array}{l}\text { Acuicultura de algas en concesiones } \\
\text { marinas }\end{array}$ & 17 \\
\hline $\begin{array}{l}\text { Buenas condiciones ambientales (nutrientes, } \\
\text { bahía protegida con aguas tranquilas, } \\
\text { diversidad de especies) }\end{array}$ & 3 \\
\hline $\begin{array}{l}\text { Mercado internacional de algas/buenos } \\
\text { precios }\end{array}$ & 14 \\
\hline Mayores fuentes de trabajo & 7 \\
\hline Apoyo de la PlusPetrol & 6 \\
\hline $\begin{array}{l}\text { Áreas de repoblamiento y extracción } \\
\text { controlada }\end{array}$ & 9 \\
\hline $\begin{array}{l}\text { Mayor colaboración entre las asociaciones, } \\
\text { con el gobierno y con las universidades }\end{array}$ & 12 \\
\hline
\end{tabular}

Al finalizar esta sección de la encuesta, los algueros y facilitadores del taller tuvieron la oportunidad de intercambiar ideas y dialogar acerca de las posibles oportunidades que las actividades de extracción de algas pudieran generar. Durante este diálogo se destacaron los siguientes temas y comentarios:

- La bahía de Pisco presenta buenas condiciones ambientales para el desarrollo y crecimiento de las algas: principalmente porque el área de Pisco tiene muchos nutrientes. Además, debido a la cercanía de las Islas Ballestas, la Bahía está protegida. La zona presenta diversidad de especies marinas.
- Viabilidad de un Proyecto de acuicultura de algas.

- Acuicultura de algas en concesiones establecidas por el gobierno.

- El mercado internacional de algas ofrece buenos precios por las algas.

- Las aguas tranquilas y los sustratos en el fondo favorecen el crecimiento de las algas.

- La calidad del agua es una interrogante y es posible que esté contaminada debido a la descarga de "agua de cola" de las empresas harineras.

- Se debe promover una mayor colaboración entre las asociaciones de algueros con el gobierno central y con las universidades interesadas en proyectos de acuicultura.

- La extracción de algas genera mayores fuentes de trabajo.

- El apoyo de la PlusPetrol favorecería la consolidación del proyecto de acuicultura.

- La varazón de algas en la costa por el mar facilita la extracción de algas.

- Se deben establecer áreas de repoblamiento de algas y la extracción de estas algas debe ser controlada.

2. Cambios medio - ambientales.

2.1¿Qué cambios ambientales observa que afectan a las algas marinas?

Escoger y marcar $(X)$ las tres (3) más importantes para la producción de algas.

\begin{tabular}{|l|c|}
\hline Contaminación del espacio marino & $\mathbf{2 1}$ \\
\hline $\begin{array}{l}\text { Disminución del recurso debido a la extracción } \\
\text { de algas en algunas áreas }\end{array}$ & 4 \\
\hline Menor cantidad de peces & $\mathbf{9}$ \\
\hline Incremento de la temperatura del agua & 3 \\
\hline Fenómenos de El Niño y La Niña & 7 \\
\hline $\begin{array}{l}\text { Reducción en la rapidez del crecimiento de } \\
\text { las algas }\end{array}$ & $\mathbf{9}$ \\
\hline Mayor crecimiento de las algas en invierno & 2 \\
\hline Cambios en el color de las algas & 5 \\
\hline $\begin{array}{l}\text { Incrementos en la temperatura del aire } \\
\text { (variabilidad/incertidumbre) }\end{array}$ & 1 \\
\hline Menos neblina y humedad & 1 \\
\hline Menor fijación de algas en el sustrato marino & 5 \\
\hline Mayor corriente de aire por temblores & 1 \\
\hline $\begin{array}{l}\text { Afloramiento de aguas con bajas temperaturas } \\
\text { y alta concentración salina }\end{array}$ & 3 \\
\hline
\end{tabular}




\subsection{Causas de contaminación del espacio marino.}

Escoger y marcar $(X)$ las tres (3) causas más importantes.

\begin{tabular}{|l|c|}
\hline Desagües de la población vertidos al mar & 13 \\
\hline $\begin{array}{l}\text { Desmonte consecuencia del terremoto } \\
\text { puestos en el mar }\end{array}$ & 1 \\
\hline $\begin{array}{l}\text { Contaminación por agua de lastre de los } \\
\text { barcos metaneros }\end{array}$ & 3 \\
\hline Polvo arrastrado por los vientos & 2 \\
\hline $\begin{array}{l}\text { Contaminación por las plantas de harina de } \\
\text { pescado }\end{array}$ & 19 \\
\hline $\begin{array}{l}\text { Otra (6 especificación no fue mencionada) } \\
\text { (1 humo de los carros) }\end{array}$ & 7 \\
\hline
\end{tabular}

2.3 Desde el año 2007 la contaminación en la bahía de Paracas - Pisco:

Marcar solamente una opción:

\begin{tabular}{|l|r|}
\hline Se ha puesto peor & $\mathbf{1 6}$ \\
\hline Ha disminuido la contaminación & 1 \\
\hline Sigue igual & 2 \\
\hline
\end{tabular}

Al finalizar esta sección de la encuesta, los algueros y facilitadores del taller tuvieron la oportunidad de intercambiar ideas y dialogar acerca de los cambios ambientales observados en la Bahía de Paracas. Se destacaron los siguientes comentarios:

- Contaminación del espacio marino: ocasionado por las operaciones de empresas existentes en la bahía, como las empresas harineras, el derrame del agua de lastre por los barcos que cargan el gas, el desagüe de los distritos de $\mathrm{Pa}-$ racas.

- Disminución del recurso (algas) debido a la depredación por parte de los extractores. Disminución del crecimiento de algas y cambio de color en las algas. (Si son verde pacae se pondrían verde amarillento).

- Incremento de temperatura del aire.

- Menos fijación en el sustrato marino.

- Cambios de temperatura del agua debido a la corriente de El niño y La Niña.

Otros temas mencionados por el expositor mas no por los algueros incluyeron:

- Menor cantidad de peces.

- Incremento de la temperatura del agua.

- Incremento del nivel del mar.
- Reducción en la rapidez del crecimiento de las algas.

- Mayor crecimiento de las algas en invierno.

- Cambios en los vientos.

- Incrementos en la temperatura del aire

- Mayor caudal del río Pisco que disminuye la salinidad.

- Menos neblina y humedad.

- Menor fijación de algas en el sustrato marino.

- Mayor corriente de aire por temblores.

- Afloramiento de aguas con bajas temperaturas y alta concentración de sales.

\section{Adaptación - acciones llevándose a cabo}

3.1 ¿Cómo han cambiado tus decisiones y actividades en respuesta a los cambios que están pasando en el medio ambiente?

Escoger y marcar $(X)$ tres (03)

\begin{tabular}{|l|c|}
\hline $\begin{array}{l}\text { Búsqueda y realización de estudios y trabajos } \\
\text { de investigación }\end{array}$ & 11 \\
\hline $\begin{array}{l}\text { Trasladándome a otras playas menos } \\
\text { contaminadas }\end{array}$ & 19 \\
\hline Participar en proyecto de acuicultura & 18 \\
\hline Se han establecido zonas de reserva & 2 \\
\hline $\begin{array}{l}\text { Mediante el cumplimiento de normas } \\
\text { nacionales (p.e sanitarias) }\end{array}$ & 3 \\
\hline $\begin{array}{l}\text { Mediante vedas para algunos tipos de } \\
\text { algas }\end{array}$ & 13 \\
\hline
\end{tabular}

3.2 ¿Qué medidas adicionales podrían ser tomadas para adaptarse al actual y al esperado cambio climático?

Escoger y marcar $(\mathrm{X})$ tres (03)

\begin{tabular}{|l|c|}
\hline Acuicultura & $\mathbf{9}$ \\
\hline Exportación & 2 \\
\hline Estudio e investigación tecnológica & $\mathbf{1 0}$ \\
\hline Extracción regulada y sostenible & 6 \\
\hline $\begin{array}{l}\text { Coordinación de los algueros con el gobierno } \\
\text { regional }\end{array}$ & 4 \\
\hline $\begin{array}{l}\text { Toma de acciones por parte del gobierno } \\
\text { regional en estos temas }\end{array}$ & 2 \\
\hline $\begin{array}{l}\text { Seminarios, cursos de entrenamiento y } \\
\text { talleres que aumenten el conocimiento }\end{array}$ & $\mathbf{1 1}$ \\
\hline Regulación y ordenamiento de la actividad & 1 \\
\hline $\begin{array}{l}\text { Vedas durante el periodo de fijación (abril y } \\
\text { mayo) }\end{array}$ & 6 \\
\hline
\end{tabular}




\begin{tabular}{|l|l|}
\hline Concesiones y zonificación marina & 7 \\
\hline Monitoreo del ambiente marino & 5 \\
\hline $\begin{array}{l}\text { Bonos de } \mathrm{CO}_{2} \text { para la protección y crecimiento } \\
\text { de las algas }\end{array}$ & 2 \\
\hline Reducción del monóxido de carbono $\left(\mathrm{CO}_{2}\right)$ & 1 \\
\hline
\end{tabular}

\subsection{Barreras a la adaptación}

Escoger y marcar $(\mathrm{X})$ tres (3)

\begin{tabular}{|l|r|}
\hline $\begin{array}{l}\text { Falta de conocimiento, información e } \\
\text { investigación }\end{array}$ & 13 \\
\hline Falta de políticas apropiadas y regulación & 5 \\
\hline $\begin{array}{l}\text { Falta de decisión y seriedad en las } \\
\text { autoridades del sector }\end{array}$ & 12 \\
\hline Falta de organización & 7 \\
\hline $\begin{array}{l}\text { Falta de colaboración e integración de } \\
\text { conocimiento }\end{array}$ & 1 \\
\hline $\begin{array}{l}\text { Falta de una coordinación integrada de los } \\
\text { recursos marinos aun con la participación de } \\
\text { la industria pesquera }\end{array}$ & 7 \\
\hline $\begin{array}{l}\text { Intereses económicos de los compradores de } \\
\text { algas }\end{array}$ & 4 \\
\hline No existen concesiones para algas & 10 \\
\hline $\begin{array}{l}\text { Extracción prohibida en áreas destinadas a } \\
\text { otros usos }\end{array}$ & 2 \\
\hline Requerimiento de mejores embarcaciones & 3 \\
\hline Contaminación marina & 5 \\
\hline
\end{tabular}

\section{Acciones futuras.}

4.1¿Qué futuro le ve usted a la industria de algas en Pisco en los próximos 10 años?

Las respuestas de los algueros se encuentran literalmente transcritas en letras itálicas.

- "Dentro de ese tiempo si continuamos en nuestro proyecto tendríamos un pueblo caminando al progreso".

- "Yo veo que esta industria de algas en Pisco, disminuirá cada vez más casi como se ve en la actualidad. Sin el apoyo de las autoridades del gobierno central y regional".

- "Veo que cada día más está disminuyendo nuestra producción. Hace 5 a 6 años atrás teníamos buena cantidad de producción donde todos los algueros trabajaban. Hoy en día ya no hay muchas algas en la playa Lobería, Puerto Nuevo, Punta Ripio, etc. Haciendo un cultivo se podría mejorar".

- "No hay futuro para las algas. La contaminación de Plus Petrol ocasiona la desaparición de las algas".
- "Yo lo miro muy difícil porque así como los vemos ahorita estamos en una situación, pero nosotros mismos podemos cambiar todo eso si ponemos todo de nosotros y de ustedes también. No se debe contaminar el mar porque no deja crecer las algas marinas y peces".

- "Si el Ministerio de Producción facilita el desarrollo del proyecto cultivo de algas mejoraría el porvenir de los algueros".

- "Así como están las algas en estos momentos con tanta contaminación no nos dan las concesiones, nos ponen mucha traba. No veo un buen futuro. Pero si trabajamos desde ahora unidos a una sola idea entre asociaciones, autoridades, empresas pesqueras, empresas mineras, gobierno regional y central, de esa forma sí llegaremos a un buen futuro promisorio".

- "En los próximos 10 años la industria de algas estaría en un caos total debido a tanta contaminación que existe en el fondo del mar, pero si se toman en cuenta programas o reglamentos que cuiden o protegen las algas, esto podría mejorar con un proyecto de algas que sería muy bueno".

- "Cada temporada las algas marinas vienen disminuyendo preocupantemente para nosotros los algueros, no teníamos este tipo de escasez anteriormente y cabe decir que el único culpable es la planta que se ha situado al frente de nuestras zonas de trabajo de extracción de algas marinas".

- "Que no hayan algas".

- "No le veo futuro porque existe mucha contaminación pero se debe tener un reglamento que regule la contaminación y protección de algas".

- "Un futuro que se haría realidad para la exportación -que como todos conocen el Perú es uno de los países abundantes de algas-, entonces cosecharíamos 6 meses y lo pondríamos en veda los otros meses, pero con un área de manejo donde no tengamos contaminación; y así sería nuestra área con resolución que nos garantizaría ante los compradores extranjeros".

- "Si no tomamos medidas adecuadas en cuanto a la conservación y manejo de dicho producto se espera un futuro incierto no solamente de algas si no de la pesca en general".

- "Si no tomamos medidas esperamos un futuro incierto".

- "Es la única alternativa en alimentos y medicinas humanas para el futuro". 
- "Si trabajamos honestamente y en equipo y es más teniendo buenas autoridades lograremos la industria de algas".

- "Creo que en 10 años no habrá algas, por todo lo que veo".

- "Si las condiciones requeridas para el cultivo de las algas y los requisitos dispuestos por el Ministerio de Producción apoyan en todo término cambiaría el futuro de los algueros de Pisco".

- "Si no tomamos la responsabilidad y las prioridades para mantenerlo, y si a ello le aumentamos los fenómenos climáticos, no le vería ningún futuro".

- "Se utiliza para la medicina".

- "Sin organización no hay futuro, pero nuestro rico mar nos da la oportunidad de progresar con ayuda".

- "Es la medicina para las personas".

4.2 ¿Qué prioridades se deberían tener en cuenta para el desarrollo futuro de esta industria?

Las respuestas de los algueros se encuentran literalmente transcritas en letra itálica.

Sobre planificación y ordenamiento de la actividad extractiva de algas:

- "En primer lugar creo que se debe priorizar: Concesiones del espacio marino para el cultivo, Apoyo del Ministerio de la Producción, Apoyo de las autoridades marítimas, Apoyo técnico y asesoramiento para el desarrollo del proyecto, Recursos para el desarrollo del proyecto".

- "Tener una planificación acerca de las algas marinas".

- "Dejar crecer las algas".

- "No sacar en exceso las algas marinas o especies".

- "Que se debería de trabajar en áreas de cultivo de algas marinas".

- "La organización en las asociaciones".

- "Ordenamiento en la recolección de algas marinas. Realizar vedas en meses de fijación".

- "Hacer repoblamiento de algas en zonas favorables y una concesión".

- "Concesiones y zonificación marina. Apoyo del Ministerio de Producción. Menos contaminación de las empresas. Seminarios y/o talleres, capacitación para la exportación. Menos contaminación por agua de lastre".
Sobre procesamiento, valor agregado y comercialización:

- "La organización y los acuerdos realizados con las plantas industriales".

- "Buena planta para proceso industrial y consumo humano".

- "La industria sería el desarrollo y nos traería mejor futuro, pues nosotros tendríamos nuestra empresa; estaríamos a nivel de Chile exportando nuestras algas ya que los mercados pesqueros son varios".

- "Necesitaría una planta adecuada para desarrollar industrialmente nuestros productos en todo término como es: blanca molida, verde deshidratada para el consumo y medicina".

- "Se puede exportar".

Sobre contaminación del espacio marino:

- "Que no haya contaminación de las fábricas".

- "No contaminar el mar".

- "Como primera prioridad sería hacer un estudio o monitoreo en áreas contaminadas luego realizar pilotos para que estas áreas nuevamente puedan repoblarse mediante una veda de 3 o 4 meses que serían suficiente para este fin".

Sobre interacción y apoyo de diferentes organizaciones:

- "Trabajar de la mano con Imarpe, Dicapi, Inrena, Produce, tener más apoyo del gobierno regional, de las universidades. Que se nos facilite concesiones y áreas de manejo para el repoblamiento de algas y sobre todo estar unidos para alcanzar la meta".

- "Más interés de parte de las autoridades gubernamentales. Conocimientos de las extracciones (charlas, talleres, etc.). Concientizar a los empresarios pesqueros (no contaminación)"

- "Apoyo del gobierno".

- "Apoyo del Ministerio de la Producción".

Sobre capacitación y aprendizaje:

- "Tener más conocimiento y con personas preparadas que nos sepan orientar y poder desarrollarnos con sabiduría y inteligencia con personas bien capacitadas".

- "Asesoramiento para el cultivo de parte de la universidad".

- "Calificar al personal que representan las asociaciones de los algueros y buscar netamente la 
organización transcurrido observadores internacionales que miren nuestros productos".

4.3 ¿Cuáles serían las mejores zonas para cultivo de algas?

Escoger y marcar $(X)$ las tres (3) mejores.

\begin{tabular}{|l|c|}
\hline Puerto Nuevo & 16 \\
\hline $\begin{array}{l}\text { Dentro de la Reserva Nacional de Paracas } \\
\text { (Mendieta p.e) }\end{array}$ & 16 \\
\hline Atenas & $\mathbf{8}$ \\
\hline Punta Ripio & 6 \\
\hline Playa San Andrés & 6 \\
\hline Playa Lobería & 5 \\
\hline Playa Santa Elena & 7 \\
\hline Playa Punta Colorada & 1 \\
\hline San Juan de Marcona & 2 \\
\hline
\end{tabular}

4.4 Otros comentarios o sugerencias que desearías agregar.

Las respuestas de los algueros se encuentran literalmente transcritas y en itálica.

- "La necesidad de un puesto de trabajo es cada vez preocupante y pienso que solo desarrollando la maricultura se podrá mitigar estas necesidades de este sector que cada vez va hacía abajo; es importante el apoyo técnico, la participación del gobierno, de las organizaciones, etc. (apoyo técnico de la Pontificia Universidad Católica)".

- "Ninguno"

- "El proyecto de algas será en beneficio de los algueros de la provincia de Pisco".

- "Que estos talleres se hagan más seguidos y que tengan una mayor difusión para que todos los compañeros tengan conocimiento y ver un local más lleno.

- "Que en el punto N. 2.2 de la contaminación marina cabe agregar el removimiento del suelo marino realizado por las Plus Petrol que nos perjudicó a todos los recolectores de algas marinas".

- "Debería haber apoyo de las empresas y un diálogo para que mejoren sus desagües o tuberías que hay en el fondo marino".

- "No hay asociación que trabaje en el cultivo de algas, los extractores no conocen de investigación o capacitación ya que la gran mayoría no tienen los conocimientos adecuados y un pequeño porcentaje ha terminado la secundaria. Desconocen hasta el nombre de las playas donde trabajan".

- "Me parece muy buena iniciativa tener el apoyo de las entidades ONG, universidades conocedoras del verdadero impacto que se origina en nuestro litoral".

- "Todas las playas señaladas en 4.3 están contaminadas menos San Juan de Marcona".

- "Otro, más capacitaciones y charlas".

- "Que deberíamos de trabajar en equipo y con buenas autoridades para el desarrollo de nuestra población".

- "Si tenemos un proyecto del cultivo de algas y concesiones para desarrollar el cultivo cambiaría la situación actual de los algueros de la provincia de Pisco".

Al finalizar esta sección de la encuesta, los algueros y facilitadores del taller tuvieron la oportunidad de intercambiar ideas y dialogar acerca de los desafíos que limitan la extracción de algas y las posibles soluciones. Se destacaron los siguientes comentarios:

\section{Desafíos}

- La contaminación del agua del mar impide el desarrollo de las algas y por consiguiente la extracción de algas es limitada.

- La cantidad de algas ha disminuido

- El establecimiento de áreas restringidas para la actividad de extracción genera conflictos sobre el espacio marino.

- La introducción de aguas calientes en la zona donde se extraen algas limita el crecimiento de éstas.

- La falta de planificación y desorganización de las asociaciones dedicadas a la extracción de algas.

- Falta de conocimiento y entrenamiento de los algueros.

- La veda de algas para la especie conocida como sargazo (Macrocystis). Se debería establecer una veda para la especie Chondrocanthus Chamissoi.

- Falta de procesamiento industrial de las algas. El proceso de algas extraídas es artesanal y por lo tanto su eficiencia es baja. 


\section{Soluciones}

- Limitar embarcaciones que se dedican a la extracción de algas.

- Trabajar organizadamente.

- Establecer una veda temporal de algas.

- Demandan el análisis de la calidad del agua a Imarpe debido a que las operaciones de empresas de harina de pescado, y de Pluspetrol contaminan el agua.

- Construcción de una laguna de oxidación para tratar aguas servidas de la población de Pisco.

\section{CONCLUSIONES Y RECOMENDACIONES}

El trabajo participativo de interacción universidad - comunidad, el cual culminó en su primera etapa con un seminario taller, obtuvo los siguientes resultados:

- Los algueros ven de una manera positiva la creación de un plan piloto para el cultivo de algas. La extracción de algas es una actividad con mucho potencial en el campo económico, que podría generar fuentes de trabajo en la zona y por consiguiente promovería el desarrollo de la bahía de Paracas.

- Las mayores dificultades que los algueros reconocen durante la extracción de algas son: la contaminación del agua de mar, la menor cantidad de algas en el mar y el establecimiento de áreas restringidas para la actividad que generan conflictos sobre el espacio marino. Los algueros atribuyen la menor cantidad de algas en el mar a la contaminación generada por las fabricas de harina de pescado (19 votos), desagües de la población vertidos al mar (13) y otras causas (7) que no fueron especificadas en su encuesta. Cabe mencionar que durante el diálogo, los algueros enfatizaron que el agua de lastre vertida al mar por los barcos transportadores de gas natural de Pluspetrol también genera contaminación.

- La contaminación del espacio marino fue el tema más discutido en durante este taller. De acuerdo con los algueros la contaminación ha disminuido la cantidad de algas y peces en el mar. Además el ciclo de crecimiento de las algas es más lento.

- Otras barreras de igual importancia para el desarrollo de las actividades extractivas de algas incluyen: 1) la falta de conocimiento o capacitación acerca del tema, 2) falta de decisión y seriedad de las autoridades del sector y 3 ) la falta de concesiones para la extracción de algas.
- De acuerdo con los algueros, las posibles medidas que ellos podrían tomar para evitar que la disminución de la cantidad de algas continúe serían: crear proyectos de cultivo de algas, organizar el trabajo de las asociaciones que extraen algas para evitar la extinción de estas, y establecer una veda temporal que permita crecimiento y reproducción adecuada de las algas.

- De acuerdo con los algueros, otras medidas a largo plazo para resolver el mencionado problema incluirían: monitorear la calidad del agua, con ayuda de Imarpe, que tendría que realizar análisis químicos. También se debería de construir una laguna de oxidación para el tratamiento de las aguas servidas generadas por la población de Pisco y por las fábricas de harina de pescado. Finalmente se debería establecer comunicación con Pluspetrol para evitar que sus barcos transportadores de gas viertan las aguas de lastre en la bahía.

- De acuerdo con los algueros, los estándares de calidad para la venta de algas en el extranjero son muy altos. Sin embargo, estos estándares no han sido descritos en detalle. Además, las previas conversaciones con los algueros sugieren que cada mercado (nacional o extranjero) tiene una preferencia para la compra de algas. Por tal motivo, para desarrollar el proyecto de cultivo de algas es necesario identificar el mercado al que se quiere vender estas algas. Consecuentemente, el proyecto de cultivo tendrá como objetivo cumplir con los estándares de control de calidad que el mercado seleccionado requiera.

- Es necesario desarrollar investigaciones científicas que permitan determinar cuáles son los parámetros óptimos para el crecimiento de las algas en Pisco. De la misma manera, es necesario realizar estudios de impacto ambiental que determinen los factores que afectan el crecimiento de las algas. Estos factores podrían ser: los efluentes de las fábricas de harina de pescado, la descarga de agua de los barcos que transportan gas natural (Pluspetrol), cambios de temperatura del agua de mar debido al cambio climático, desagües vertidos al mar, desmontes de concreto generados por el terremoto en el 2007, relaves mineros, pesticidas usados en la agricultura, etc.

- Todos los estudios antes mencionados son indispensables para el desarrollo de un plan piloto para el cultivo de algas. Sin embargo, estos estudios requerirán financiamiento económico. Se espera contar, en un futuro cercano, con la cola- 
boración económica de entidades como Imarpe, Concytec o Pluspetrol.

A partir de estas conclusiones se recomienda una acción participativa del tipo triple hélice: universidad - gobierno - empresa en San Andrés y en San Clemente alrededor de la temática de las algas marinas.

Impulsar el otorgamiento de concesiones a las asociaciones de algueros para el cultivo de algas paralelamente con la capacitación en técnicas de cultivo.

Evaluar e implementar soluciones sistémicas a la contaminación del espacio marino mediante acciones concretas como el de la laguna de oxidación.

Introducir mejores prácticas de manufactura y trazabilidad en el procesamiento de las algas marinas, implementando mejoras en el lavado y el secado.

Transferir conocimiento y realizar capacitaciones en asociatividad, técnicas de cultivo y procesamiento.

Realizar investigaciones que busquen mejoras tecnológicas en técnicas de cultivo, así como darle valor agregado a las algas marinas de la provincia de Pisco.

\section{REFERENCIAS BIBLIOGRÁFICAS}

[1] Álvarez, J.C; Vodden, K. (2009). Local Ecological Knowledge and the Impacts of Global Climatic Change on the Community of Seaweed Extractors in Pisco-Perú. Portland International Center for Management of Engineering and Technology PICMET'09. Portland, Estados Unidos de América.

[2] Asociación de productores de harina de pescado. (2007). Manejo y disposición de efluentes. http:// www.apropisco.org/index.php?option=com_co ntent\&view=article\&id=10\&ltemid=4 (visitado el 05-04-2011).

[3] Dirección de Hidrografía y Navegación de la Marina de Guerra del Perú (2007). Fenómeno de El Niño. http://www.dhn.mil.pe/docs/elnino/fenomeno_el_nino.pdf (visitado el 05-04-2011)

[4] Enfield, D. (2003). The "El Niño" FAQ: Frequently Asked Questions About El Niño-Southern Oscillation (ENSO). http://www.aoml.noaa.gov/general/enso_faq/ (visitado el 05-04-2011)

[5] Imarpe. (2009). Imarpe inició investigaciones para la obtención de biocombustible a base de algas marinas. http://www.imarpe.pe/imarpe/ index.php?id_detalle $=00000000000000008451$ (visitado el 05-04-2011)

[6] INEI (2007) Censo de Población y Vivienda 2007.

[7] ProParacas. (2007). Comisión para el desarrollo sostenible de la Bahía de Paracas.

www.labor.org.pe/descargas/Foro\%2010-0206,\%20Proparacas.ppt (visitado el 05-04-2011)

[8] Pulache, W. (2010). Vientos extremos en la costa peruana, causas y efectos. Simpósio: Eventos hidrometereológicos extremos y cambio climático en el Peru. Ministerio del Ambiente Senhami. http://www.senamhi.gob.pe/?p=0901 (visitado el 6 de abril del 2011)

[9] Universidad del Pacífico (1999). Spices and Herbs A SURVEY OF THE NETHERLANDS. http:// www.up.edu.pe/carrera/administracion/SiteAssets/Lists/JER_Jerarquia/EditForm/2spicesh. pdf (visitado el 06-04-2011) 